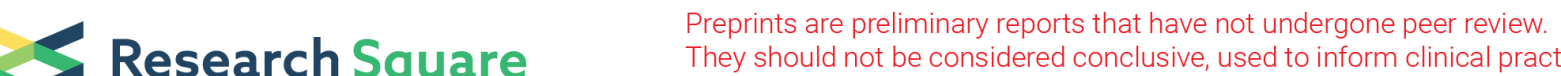 Research Square They should not be considered conclusive, used to inform clinical practice, or referenced by the media as validated information.
}

\section{Melatonin Protects TEGDMA induced Pre- odontoblasts Mitochondrial Apoptosis via JNK/MAPK Signaling Pathway}

Qihao Yu

School \& Hospital of Stomatology Wenzhou Medical University

Yi Liu

Sichuan University West China Hospital of Stomatology: Sichuan University West China College of

Stomatology

Konghuai Wang

School \& Hospital of Stomatology Wenzhou Medical University

Xunben Weng

School \& Hospital of Stomatology Wenzhou Medical University

Shengbin Huang

Affiliated hospital of Xi'an Medical University: Shaanxi Provincial People's Hospital

Yihuai Pan ( $\boldsymbol{\nabla} 773502228 @ q q . c o m$ )

School \& Hospital of Stomatology Wenzhou Medical University https://orcid.org/0000-0001-61624165

\section{Research Article}

Keywords: TEGDMA, Apoptosis, Mitochondrial dysfunction, Dental pulp cell, Melatonin, MAPK

Posted Date: August 24th, 2021

DOl: https://doi.org/10.21203/rs.3.rs-779368/v1

License: (a) (1) This work is licensed under a Creative Commons Attribution 4.0 International License. Read Full License 


\section{Abstract}

Background: Resin monomer induced dental pulp injury presents a mitochondrial dysfunction related pathology. Melatonin has been regarded as a strong mitochondrial protective bioactive compound from pineal gland. However, it remains unknown whether melatonin can prevent dental pulp from resin monomer induced injury. The aim of the study is to investigate the effects of melatonin on TEGDMA, a major component in dental resin, induced mouse pre-odontoblast cell lines (mDPC6T) mitochondrial apoptosis and to verify whether JNK/MAPK signaling pathway mediate the protective effect of melatonin.

Methods: We adopted a well-established TEGDMA-induced mDPC6T apoptosis model to investigate the preventive effect of melatonin by detecting cell viability, apoptosis rate, expression of apoptosis related protein, mitochondrial ROS (mtROS) production, mitochondrial membrane potential (MMP) and adenosine triphosphate (ATP) level. Inhibitors of MAPKs signaling were used to explore which pathway was participated in TEGDMA induced apoptosis. Finally, we verified the role of JNK/MAPK pathway during the protective effects of melatonin above by the agonist and antagonists of JNK.

Results: Melatonin attenuated TEGDMA induced mDPC6T apoptosis via reducing mtROS production, rescuing MMP and ATP level. Meanwhile, the mitochondrial dysfunction and apoptosis was alleviated by the JNK/MAPK inhibitor SP600125 but not the other MAPKs signaling inhibitors. Furthermore, melatonin down-regulated the expression of phosphorylated-JNK, and eliminated the active effects of Anisomycin on JNK/MAPK pathway, which mimicked the effects of the SP600125.

Conclusion: Our findings demonstrated that melatonin protected mDPC6T against TEGDMA induced apoptosis via JNK/MAPK signaling and maintenance of mitochondrial function, which presented a novel therapeutic strategy for prevention against resin monomer-induced dental pulp injury.

\section{Background}

Due to the superior performance, easy-to-operate and aesthetic properties, resin containing compounds are being used for a wide variety of dental applications, such as restorations, sealants, bonding agents, and pulp capping ${ }^{[1][2]}$. Among the most common used monomer compounds in dental resins are bisphenol-A-glycidyl methacrylate (Bis-GMA), 2-hydroxyethyl methacrylate (HEMA) and triethylene glycol dimethacrylate (TEGDMA $)^{[3]}$. Depending on the application, varying amounts of the above monomers do not polymerize and would leach out of resin monomer to oral tissues ${ }^{[4]}$. These resin monomers, such as TEGDMA, elicit a wide variety of oral tissue cell responses including apoptosis, impairment of mineralization and disturbance of functions. However, as the common presentation of cell toxicity, apoptosis presents the main manifestation of resin monomer-induced dental pulp injury ${ }^{[5] ~[6] ~[7] ~[8] ~}$

Mitochondria play vital roles in many cellular processes including cell proliferation, metabolism and apoptosis $^{[9][10][11]}$. Accumulating evidence has implicated excess reactive oxygen species (ROS) in 
mitochondria promote caspase-dependent apoptosis, so mitochondria are identified as the center of apoptosis through the intrinsic pathway ${ }^{[12]}$. Furthermore, Redundant ROS occurs respiratory dysfunction and reduces adenosine triphosphate (ATP) generation, which furtherly promote mitochondrial ROS ( $m$ tROS) production and oxidative damage, therefore vicious circle forms. Previous researches showed the cytotoxicity of TEGDMA to oral tissues involves in accumulating mtROS and irreversible mitochondria damage $^{[13][14]}$. Our latest study has also demonstrated that mitochondrial dysfunction was the major factors in the pre-odontoblasts apoptosis induced by TEGDMA ${ }^{[15]}$. However, the detailed mechanism, especially the signaling pathway to regulate the mitochondrial dysfunction in TEGDMA induced cell apoptosis need to be further explored.

The mitogen-activated protein kinases (MAPKs) pathway, which are grouped into distinct families (the extracellular signal-regulated protein kinases1/2 (ERK1/2), the c-Jun N-terminal kinases (JNK) and the p38 MAPKs), have been proven to be essential for controlling diverse cellular behaviors including cell proliferation, differentiation and apoptosis ${ }^{[16]}[17]$. It's noted that he role of the MAPKs pathway on mitochondria focused on the phosphorylation of executive function protein that are essential to fundamental mitochondrial functions in energy production, redox processes and metabolic pathways ${ }^{[18]}$. Meanwhile, the sub-families of MAPKs present different function on mitochondria. JNK has been implicated in the generation of high amounts of mtROS when it translocated to the mitochondria ${ }^{[19]}$, ERK $1 / 2$ and p38 act as upstream signal of mitochondria membrane potential disruption, cytochrome $c$ release, and caspase- 3 activation ${ }^{[20]}$. On the other hand, in resin monomer-induced mouse macrophages apoptosis, the activated ERK/JNK/p38 MAPKs induced by TEGDMA was inhibited by antioxidant Nacetylcysteine (NAC) ${ }^{[21]}$. Another study pointed out that it was $\mathrm{p} 38$ and ERK1/2, but not JNK, participated in TEGDMA induced cell apoptosis ${ }^{[22]}$. Therefore, it needs to be further clarified that which MAPKs pathway is involved in the TEGDMA-induced pre-odontoblasts mitochondrial apoptosis, as well as finding a mitochondria-targeted antioxidant which able to effectively sequester mtROS and protect against mitochondrial damage by the mechanism.

Melatonin synthesizes and secrets mainly by the pineal gland during the alternations of light and darkness. Similarly, melatonin is commonly ingested from drugs, serving as an oral mitochondrial protective drug ${ }^{[23]}$. Previously, it was shown that melatonin was employed in fields of oral diseases and material applications. For instance, melatonin has been shown to relieve the symptoms of dental pulpitis ${ }^{[24]}$ and stimulate osteointegration of dental implants ${ }^{[25]}$. Meanwhile, as an indoleamine, melatonin might act as the new possible application to improve properties of biomaterials used in dentistry ${ }^{[26]}$. As a strong mitochondrial protective substance, melatonin enhances mitochondrial electron transfer chain (ETC) Complex I and IV, thereby improving mitochondrial respiration, ATP synthesis and energy metabolism under stress circumstances ${ }^{[27]}$. Evidences suggested that melatonin attenuated apoptosis induced by $\mathrm{H}_{2} \mathrm{O}_{2}$ in human dental pulp cells (hDPCs) ${ }^{[28][29]}$. Physiological concentrations of melatonin can inhibit proliferation and promote odontogenic differentiation of hDPCs ${ }^{[30]}{ }^{[31]}$. There has been announced that exogenous melatonin can improve the oxidative stress damage mediated by 
MAPKs pathway ${ }^{[32]}$, but its special effect on dental pulp cells apoptosis and mitochondrial damage induced by TEGNMA remains unknown.

In this study, we adopted a well-established vitro TEGDMA induced pre-odontoblast cells apoptosis model, aiming to 1) investigate the effect of melatonin on TEGDMA-induced pre-odontoblasts mitochondrial dysfunction and apoptosis; 2) clarify the role of MAPKs signaling pathway on the protective effect of melatonin.

\section{Methods And Materials \\ 2.1. Reagents}

Cell culture medium and supplements were purchased from Life Technologies (Grand Island, NY, USA). Triethylene glycol dimethacrylate (TEGDMA; CAS-No. 109-16-0), SP600125 (\#S5567), 3-(4,5-dimethylthiazol-2-yl)-2,5-diphenyltetrazolium bromide (MTT) (\#M2128), melatonin (\#M5250), SP600125(\#S5567), PD98059(\#P215) and SB203580(\#S8307) were obtained from Sigma-Aldrich (St. Louis, MO, USA). Antibodies against phosphorylated JNK (Thr183/Tyr185) (p-JNK) (\#4668S), JNK (\#9252S), phosphorylated ERK1/2 (Thr202/Tyr204) (p-ERK1/2) (\#4370S), ERK1/2 (\#4969S), phosphorylated p38 (Thr180/Tyr182) (p-p38) (\#4511S), p38 (\#8690S), Bax (\#2772S), Bcl-2(\#15071), Caspase3 (\#9662S) and $\beta$-actin (\#3700) were obtained from Cell Signaling Technology (Beverly, MA, USA). Annexin V-fluorescein isothiocyanate (FITC) apoptosis detection kit, Chamber slides, goat antirabbit (\#656120) and anti-mouse (\#626520) secondary antibodies, 4',6-diamidino-2-phenylindole (DAPI) (\#P36931) were obtained from Invitrogen (Carlsbad, CA, USA). MitoSOX Red (\#M36008), MitoTracker Green (Mitogreen) (\#M7514) and tetramethylrhodamine methyl ester (TMRM) (\#T668) were purchased from Life Technologies. Terminal deoxynucleotidyl transferase dUTP nick end labeling (TUNEL) assay kits (\#11684795910) were obtained from Roche (Mannheim, Germany). ATP assay kits (\#S0026) were obtained from Beyotime (Shanghai, China). Anisomycin (\#S7409) was obtained from Selleckchem (Shanghai, China).

\subsection{Cell Culture}

mDPC6T cells, a pre-odontoblast cell lines, were the gift from the Prof. Chen Zhi (Wuhan University, Wuhan, PR China). Cells were maintained in Dulbecco's Modified Eagle's Medium (DMEM) supplemented with $10 \%$ fetal bovine serum and antibiotics (100 IU/ml penicillin $\mathrm{G}$ and $100 \mathrm{ng} / \mathrm{ml}$ streptomycin) in a humidified incubator at $37^{\circ} \mathrm{C}$ with $5 \% \mathrm{CO}_{2}$.

\subsection{Cell Treatments}

Test compounds were prepared as stock solutions and diluted to the desired final concentrations immediately before use. Final concentrations of the compounds were as follows: TEGDMA (2 mM), SP600125 $(10 \mu \mathrm{M})$ and Anisomycin $(1 \mu \mathrm{M})$. The final concentration of dimethyl sulfoxide (DMSO) in the 
culture was less than $0.5 \%$ in all experiments. Cells were treated with or without TEGDMA and the indicated test compounds for various times, according to the experimental protocol.

\subsection{Cell Viability Test}

mDPC6T cells were seeded in 96-well plates $\left(1 \times 10^{4}\right.$ cells/well) and cultured under different conditions, as indicated for each experiment. Briefly, mDPC6T cells were washed twice with phosphate buffered saline (PBS), and incubated in $100 \mu \mathrm{l} /$ well serum-free medium supplemented with $20 \mu \mathrm{L}$ MTT solution (5 $\mathrm{mg} / \mathrm{ml}$ ) at $37^{\circ} \mathrm{C}$. After 6 hours, the supernatant was removed, and the formazan crystals were dissolved in $100 \mu \mathrm{L} /$ well of DMSO for $10 \mathrm{~min}$. The plates were then agitated for $15 \mathrm{~s}$, and the absorbance was measured at $570 \mathrm{~nm}$ using a microplate reader.

\subsection{Apoptosis Measurement by Flow Cytometry}

Flow cytometry was performed to identify the cell cycle and apoptotic cells. Annexin-V labeled with fluorescein isothiocyanate and propidium iodide $(\mathrm{Pl}, 1 \mu \mathrm{g} / \mathrm{ml})$ was used to determine cell apoptosis and necrosis. After exposure to various experimental conditions, cells were trypsinized, and labeled with fluorochromes at $37^{\circ} \mathrm{C}$, and then cytofluorometric analysis was performed with a FACScan (Becton Dickinson, NY, USA).

\subsection{TUNEL Assays}

TUNEL staining was carried out to identify the rate of apoptotic cells. For the TUNEL assays, cells inoculated on a coverslip were fixed in $4 \%$ paraformaldehyde (PFA) in PBS, and permeabilized with $0.2 \%$ Triton X-100 in citrate buffer. Samples were incubated with TUNEL reaction mixture at $37^{\circ} \mathrm{C}$ for 1 hour, counterstained with DAPI, and observed with a fluorescence microscope (Leica TCS SPE, Germany). The percentage of apoptotic cells was estimated by counting a total of 300 cells from random fields.

\subsection{Western Blot Analyses}

After the indicated treatments, cells were collected and lysed in cell lysis buffer (Cell Signaling Technology, Beverly, MA, USA). Protein concentrations were determined using a Bradford protein assay kit (Thermo Fisher Scientific). Proteins were separated by electrophoresis and transferred to a polyvinylidene difluoride (PVDF) membrane. Anti-p-JNK (1:2000), anti-JNK (1:2000), anti-ERK1/2 (1:2000), anti-p-ERK1/2 (1:2000), anti-p-p38 (1:2000), anti-p38 (1:2000), anti-Bax (1:2000), anti-Bcl-2(1:2000), anti-Caspase3 $(1: 1000)$, and anti- $\beta$-actin $(1: 8000)$ antibodies were used as primary antibodies. Proteins bound by primary antibodies were visualized with an appropriate secondary antibody (1:4000), followed by the addition of an enhanced chemiluminescence substrate (Thermo Fisher Scientific). The protein bands were detected using a Bio-Rad imaging system (Bio-Rad, Hercules, CA, USA) and quantified with NIH ImageJ software.

\subsection{Mitochondrial Functional Imaging Assays}

Cells were seeded in chamber slides at $1 \times 10^{4}$ cells/well. Cells were treated with TEGDMA and other test compounds for $1 \mathrm{~h}$. Then, the cells were incubated in fresh culture medium containing $2 \mu \mathrm{M}$ MitoSOX for 
30 mins. To assess the MMP, cells were co-stained with Mitogreen (100 nM) and TMRM (100 nM) for 30 min, according to our previous work ${ }^{[15]}$, Images were captured under a fluorescence microscope.

Excitation wavelengths were $543 \mathrm{~nm}$ for MitoSOX and TMRM and $488 \mathrm{~nm}$ for Mitogreen. Post-acquisition processing was performed with $\mathrm{NIH}$ ImageJ software to measure and quantify fluorescence signals. Mitochondrial fluorescence intensities, density (NIH ImageJ software), and length (ImageJ software) were quantified by an investigator blinded to the experimental group. More than 30 clearly identifiable mitochondria in 10-15 randomly selected cells per experiment were measured according to our previous studies.

\subsection{ATP Detection}

For measurement of ATP level, whole-cell extracts from indicated cells were lysed in lysis buffer provided in the ATP Assay Kit. After centrifuged at $12,000 \mathrm{~g}$ for $5 \mathrm{~min}$ at $4^{\circ} \mathrm{C}$, the supernatants were transferred to a new $1.5 \mathrm{ml}$ tube for ATP test. The luminescence from a $100 \mu \mathrm{l}$ sample was assayed in a luminometer (Molecular Devices) together with $100 \mu$ of ATP detection buffer. The standard curve of ATP concentration was prepared from a known amount $(1 \mathrm{nM}$ to $1 \mu \mathrm{M})$. All the experiments were carried out with triplicates. Values reported are means of three replicates \pm the standard deviation (SD) of mean.

\subsection{Data Analysis}

Data were presented as mean \pm SD. Statistical analysis was performed using Statview software (SAS Institute, Version 5.0.1). For comparisons between multiple groups, one-way ANOVA was used followed by individual post hoc Fisher tests when applicable. $p<0.05$ was considered statistically significant.

\section{Results}

\subsection{TEGDMA reduced cell viability and induced apoptosis in $\mathrm{MDPC6T}$.}

Firstly, we performed MTT assays to determine the cytotoxic effect of TEGDMA on mDPC6T cells. TEGDMA in the concentrations ranging from $1 \mathrm{mM}$ to $4 \mathrm{mM}$ significantly had cell viability decreased in a time- and dose-dependent manner (Fig. 1A). The median lethal dose of TEGDMA treatment was $2 \mathrm{mM}$ after a 6 -h treatment. Consequently, we used $2 \mathrm{mM}$ in the following experiments. Flow cytometric analysis showed increase of cell apoptosis, mainly in the form of early apoptosis (Fig. 1B-C). TEGDMAinduced DNA damage was further confirmed by a significantly increased TUNEL-positive staining (Fig. 1D-E). The expression changes of apoptosis-related proteins in TEGDMA-induced mDPC6T cells apoptosis were examined by immunoblot analyses (Fig. 1F-I). TEGDMA significantly increased the expression of the apoptosis-related protein Bax, but the anti-apoptosis-related protein Bcl-2 not changed. Furthermore, compared with control, TEGDMA significantly stimulated the expression of cleavedCaspase3 in mDPC6T cells by 7.9 times. These results indicated that TEGDMA-induced cell death occurred primarily through apoptosis in mDPC6T cells. 


\subsection{TEGDMA induced mitochondrial dysfunction in mDPC6T cells.}

To identify whether mitochondrial dysfunction was involved in the TEGDMA-induced mDPC6T cells apoptosis, we evaluated mitochondrial function through the detection of mIROS, MMP and ATP. TEGDMA caused mitochondrial oxidative stress, indicated by the significantly higher staining intensity of MitoSOX (Fig. 2A-B). In addition, MMP was significantly down-regulated in the TEGDMA-treated cells (Fig. 2C-D). Moreover, the ATP level, a major indicator of mitochondrial energy-generating function, was significantly reduced in TGDMA-treated mDPC6T cells (Fig. 2E). All these results showed that TEGDMA-induced mDPC6T cells apoptosis was associated with mitochondrial dysfunction. Collectively, these results proved that TEGDMA-induced mDPC6T cells apoptosis was related to mitochondrial oxidative and mitochondrial dysfunction.

\subsection{Melatonin attenuated TEGDMA-induced apoptosis and mitochondrial dysfunction in mDPC6T cells.}

Melatonin, an active circadian regulator secreted by the pituitary gland and distributed throughout the body ${ }^{[23]}$, including the oral tissues, has been shown to have a strong mitochondrial-targeted antioxidant and anti-apoptotic effects on various types of cells. To explore the effect of melatonin in TEGDMA induced mDPC6T cells apoptosis, we treated mDPC6T cells with melatonin 1 hour prior to TEGDMA exposure. As shown in Fig. 3A, melatonin partially rescued viability of mDPC6T cells. Moreover, the DNA damage and apoptosis induced by TEGDMA was significantly ameliorated by melatonin as reflected by flow cytometry (Fig. 3B-C). Melatonin also down-regulated the expression levels of Bax and cleavedCaspase3 induced by TEGDMA (Fig. 3D-F). These data indicated that melatonin attenuated TEGDMAinduced mDPC6T cells apoptosis. In addition, melatonin significantly ameliorated the mitochondrial OS levels, as indicated by reduced MitoSOX staining intensity (Fig. 4C-D). Compared with TEGDMA alone, melatonin improved MMP levels, as shown by increased TMRM intensity (Fig. 4A-B). In addition, we evaluated the effects of melatonin on mitochondrial energy-producing function. As results showed, compared with TEGDMA, melatonin rescued the cellular ATP level (Fig. 4E). In summary, melatonin ameliorated TEGDMA-induced apoptosis and mitochondrial dysfunction in mDPC6T cells.

\subsection{JNK/MAPK pathway involved in TEGDMA induced mDPC6T mitochondrial apoptosis}

We next analyzed whether TEGDMA-induced mDPC6T apoptosis and mitochondrial dysfunction involves MAPKs family proteins, including p38, JNK and ERK1/2. We used MitoQ, a classic mitochondrial function protectant, to explore the role of the MAPKs pathways in the model. The results demonstrated that stimulation of mDPC6T cells with TEGDMA could promote the phosphorylation level of JNK phosphorylation levels, but not the p38 and ERK1/2 phosphorylation levels (Fig. 5A-D). Meanwhile, pretreatment of MitoQ reduced the phosphorylation level of JNK, but didn't affect the other MAPKs pathways (Fig. 5A-D). In order to further verify the role of MAPKs signaling pathway, we next used 
chemical inhibitors of MAPKs, SP600125, PD98059 and SB203580, which specifically inhibit the actions of JNK, ERK1/2 and p38, to examined the ability to rescue TEGDMA-induced mDPC6T cells apoptosis. MTT assays determined that only the SP600125 protected the cytotoxic effect of TEGDMA on mDPC6T (Fig. 5E-G). At the same time, cell flow cytometry revealed the reversal of SP600125 on apoptotic cells, but the reversal effects of PD98059 and SB203580 were not obvious (Fig. 5H-I). Then, Western Blot showed SP600125 reduced the protein expression of cleaved-Caspase3 and Bax (Fig. 6A-D), indicating that SP600125 protected mDPC6T cells from TEGDMA-induced apoptosis. Next, we furtherly determined the role of JNK/MAPK signaling on TEGDMA-induced mitochondrial dysfunction. We found that SP600125 ameliorated mDPC6T mitochondrial dysfunction, as evidenced by decreased mtROS production (Fig. 6E-F), increased MMP (Fig. 6G-H) and cellular ATP level (Fig. 6I). In summary, above results provided direct evidence that JNK/MAPK pathway significantly participate in TEGDMA-induced mDPC6T mitochondrial apoptosis.

\subsection{Melatonin prevent TEGDMA-induced apoptosis through JNK/MAPK pathway}

We finally explored whether melatonin involved JNK/MAPK pathway to protect TEGDMA-induced apoptosis. The JNK agonist Anisomycin increased the level of cell apoptosis by about $15 \%$ compared with TEGDMA alone, and melatonin could effectively inhibit the apoptosis of this part to normal levels (Fig. 7A-B). Western Blot results proved that melatonin reduced the level of p-JNK and reverse the further phosphorylation activation of JNK induced by Anisomycin (Fig. 7C-F). At the same time, the results of apoptotic-related protein immunoblotting showed that melatonin restored the increase of cleavedCaspase3 and Bax caused by Anisomycin (Fig. 7C-F). At the same time, Moreover, the increase of aggravated mtROS (Fig. 8A-B), the decrease of MMP (Fig. 8C-D), and the decrease of ATP production (Fig. $8 \mathrm{E})$ are restored to the damage degree induced by TEGDMA with Anisomycin through the pretreatment of melatonin. This suggests that melatonin alleviates mitochondrial dysfunction and cell apoptosis caused by TEGDMA by inhibiting the JNK/MPK pathway.

\section{Discussion}

The apoptosis is the main form of common resin composite materials induced dental pulp injury. Melatonin, a physiologically secreted anti-oxidant and mitochondrial protective product, able to efficaciously ameliorate mitochondrial dysfunction and inhibit cell apoptosis. However, there have no report on the role of melatonin in anti-resin monomer damage and its potential protective mechanisms. In our study, for the first time, we focus on the MAPK signaling, especially the JNK/MAPK pathway on the TEGDMA caused pre-odontoblasts mitochondrial apoptosis, and announce the pharmaceutical protection of the melatonin therefore representing a promising approach to the management of resin monomer induced dental pulp injury.

MtROS is an widely accepted initiator of apoptosis through numerous mechanisms, and it is not only related to damage of mitochondrial function, but also leading to an apoptotic signaling pathway 
cascade $^{[9][33][34]}$. Notably, as the products of mitochondrial metabolism, mtROS comes from the respiratory chain when mitochondria are functionally disordered ${ }^{[12]}$. Mikulás et.al have found that TEGDMA inhibited Complex I in the respiratory chains of mitochondria isolated from guinea pig brain ${ }^{[35]}$. Nonetheless, our research demonstrated that TEGDMA damaged the Complex III activity of preodontoblasts, the disordered mitochondria manifested as ATP synthesis decrease and mtROS production increase $^{[15]}$. Furthermore, mtROS generation is believed to be dependent on membrane potential of mitochondria. As the essential storage pool for electron chain, damaged mitochondria promote a reduction in electron flow when membrane potential across the inner membrane lost, hence ROS produce. As expected, our results found that the level of MMP and ATP significantly decreased, and the mtROS level was up-regulated, which confirmed the role of compromised mitochondrial function in TEGDMAinduced mDPC6T cells apoptosis.

Melatonin, a neurohormone mainly produced by the pineal gland, is a pleiotropic molecule with diverse physiological functions. In addition to its time-keeping function, melatonin acts as a potential mtROS scavenger and exhibits effective antioxidant properties. Studies have showed that this versatile hormonal compound has well-established anti-apoptotic activities ${ }^{[36]}\left[{ }^{[37]}\right.$. However, there is no report on the protective effect of melatonin on TEGDMA induced pro-odontoblasts apoptosis as well as its action mechanism. Here, we found that pretreatment with melatonin effectively inhibited TEGDMA-induced apoptosis of mDPC6T cells by mitigating the mitochondrial dysfunction. Notably, melatonin may act on cells through the following mechanisms. Firstly, because of its highly lipophilic nature, melatonin can penetrate cellular and membrane structure, and finally accumulates in mitochondria ${ }^{[38]}$. While it will enhance the activity of catalase, decreases $\mathrm{Ca}^{2+}$ influx, eliminates residual mtROS and maintains mitochondrial function ${ }^{[39]}$. On the other hand, the second way is that melatonin regulates the program cell death through a melatonin receptor. The G protein-coupled membrane receptors MT1 and MT2 are considered the primary molecules mediating the receptor-dependent pathways of melatonin ${ }^{[40]}{ }^{[41]}$. MT1 and MT2 trigger several signaling pathways, including the cAMP-response element binding protein (CREB), phosphatidylinositol 3-kinase (PI3K) and MAPKs signal pathways, and integrate different linear inputs to regulate cellular functions, such as circadian rhythm, cell differentiation, cumulus expansion and cell programed death ${ }^{[42]}[43][44]$. However, which pathway is involved in the protective effect of melatonin on pro-odontoblastic cell apoptosis needs further verification.

Activated MAPKs exert various biological effects by promoting the phosphorylation of downstream substrates which then serve as signals in various cell responses including apoptosis. In our study, the phosphorylation of MAPKs was detected by Western blot, only p-JNK changed with time and concentration when mDPC6T was stimulated by TEGDMA. The results are different from those of previous studies ${ }^{[21] ~[22]}$, as they found the phosphorylation of ERK1/2 and p38 MAPKs were more pronounced. Previous researches studied the effect of TEGDMA in mouse macrophages and treated cells for 24-48 hours, but we stimulated the mDPC6T with TEGDMA for about 6 hours. The distinct cell types and processing conditions may contribute to those inconsistences. Therefore, we specifically focused on JNK pathway regulated apoptosis. As expected, pretreatment with SP600125 notably abolished 
TEGDMA-induced apoptosis, increased mitochondrial function and suppressed mtROS production, and the opposite effect of Anisomycin on these results further proved that the JNK pathway is involved in the apoptotic effect of TEGDMA. JNK/MAPK not only is required for the release of Cytochrome $C$ from the inner membrane space of mitochondria and the activation of the pro-apoptosis protein, like Bax and Caspase- ${ }^{[45]}$, but also leads to inhibition of mitochondrial respiration and electron transport, and the damaged mitochondria leads to the release of $\mathrm{mtROS}$ and the improvement of MMP ${ }^{[46]}$. Mechanistically, the directly disrupt of the interaction between JNK and mitochondria plays the important role in

apoptosis. What's more, Schweikl et.al ${ }^{[3]}$ have reviewed the influence of monomer-induced oxidative stress on central signal transduction pathways including JNK/MAPK, which further confirmed that JNK singling pathway plays an important activator in TEGDMA-induced cell apoptosis. However, the pivotal role of JNK requires further validation through overexpression or silence by siRNA in our future study.

The release of melatonin in response to cellular stress by activating the JNK/MPAK pathway has been reported in various pathological processes ${ }^{[29][42]}$. In our study, we found that melatonin antagonized mtROS and mDPC6T cells apoptosis caused by TEGDMA or Anisomycin alone. Meanwhile, melatonin significantly inhibited cell apoptosis induced by TEGDMA and Anisomycin together. Furthermore, melatonin mimicked the effects of the inhibitor SP600125 and abolished the suppressive effects of TEGDMA on p-JNK. Therefore, we proposed that melatonin played the role of mitigating mitochondrial dysfunction regulated apoptosis partly through the JNK/MAPK pathway. However, whether the receptors MT1 and MT2 act as upstream of JNK pathway in this process deserve further investigation.

Our current study, for the first time, demonstrated that melatonin exhibited excellent protective effect on pre-odontoblast cell apoptosis induced by TEGAMA, but there are still some limitations in the experiment. On one hand, a mouse pre-odontoblast cell line was only adopted, the primary dental pulp cell is needed to confirm the mechanism underlying the protective effect of melatonin on TEGDMA induced apoptosis. On the other hand, further in vivo studies should be performed to verify the preventive effect of TEGDMA on dental pulp injury and corroborate the role of JNK/MAPK signaling pathway.

\section{Conclusion}

In summary, JNK/MAPK signaling pathway may be the key mechanism responsible for melatonin's protection effect of TEGDMA induced mitochondrial apoptosis of mDPC6T cells (Fig.9). Melatonin could act as a potential therapeutic agent for the prevention or treatment of resin monomer induced dental pulp damage.

\section{Abbreviations}

ROS: Reactive Oxygen Species; mtROS: Mitochondrial ROS; MMP: Mitochondrial Membrane Potential; ATP: Adenosine Triphosphate; TEGDMA: Triethylene Glycol Dimethacrylate; JNK: C-Jun N-terminal Kinase; MAPK: Mitogen-activated Protein Kinase; ERK1/2: Extracellular Regulated Protein Kinases 1/2; HEMA: 2hydroxyethyl Methacrylate; Bis-GMA: Bisphenol-A-glycidyl Methacrylate; NAC: N-acetylcysteine; hDPCs: 
Human Dental Pulp Cells; TMRM: Tetramethylrhodamine Methyl Ester; TUNEL: Terminal Deoxynucleotidyl Transferase dUTP Nick End Labeling; DAPI: 4',6-diamidino-2-phenylindole

\section{Declarations}

\section{Acknowledgements}

Not applicable.

\section{Authors' contributions}

Qihao Yu: contributed to design, data acquisition and analysis. Yi Liu: contributed to draft and critically revise the manuscript. Konghuai Wang and Xunben Weng: contributed to design, data acquisition and critically revised the manuscript. Shengbin Huang: contributed to critically revised the manuscript. Yihuai Pan: contributed to design and critically revised the manuscript. All authors have read and approved the final manuscript.

\section{Funding}

The work was supported by Zhejiang Provincial Natural Science Foundation of China (\#GF21H140019) to Yihuai Pan

\section{Availability of data and materials}

Not applicable.

\section{Ethics approval and consent to participate}

Not applicable.

\section{Consent for publication}

Not applicable.

\section{Competing interests}

The authors declared no conflict of interest. 


\section{Author details}

${ }^{1}$ Department of Endodontics, School and Hospital of Stomatology, Wenzhou Medical University, Wenzhou 325027, PR China. ${ }^{2}$ Institute of Stomatology, School and Hospital of Stomatology, Wenzhou Medical University, Wenzhou 325027, PR China. ${ }^{3}$ State Key Laboratory of Oral Disease, National Clinical Research Center for Oral Disease, West China Hospital of Stomatology, Sichuan University, Chengdu 610041, PR China. ${ }^{4}$ Department of Prosthodontics, School and Hospital of Stomatology, Wenzhou Medical University, Wenzhou 325027, PR China.

\section{References}

1. Pratap B, Gupta RK, Bhardwaj B, Nag M. Resin based restorative dental materials: characteristics and future perspectives. Jpn Dent Sci Rev. 2019;55(1):126-38.

2. Nakayama Wt Fau DR - Hall DE, Hall Dr Fau - Grenoble JL, Grenoble De Fau - Katz, JL, Katz. Elastic properties of dental resin restorative materials, J Dent Res 53(5) (1974):1121-6.

3. Krifka S, Spagnuolo G, Schmalz G, Schweikl H. A review of adaptive mechanisms in cell responses towards oxidative stress caused by dental resin monomers. Biomaterials. 2013;34(19):4555-63.

4. Van Landuyt KL, Nawrot B, Fau - T, Geebelen J, Geebelen B, Fau - De Munck, J, De Munck JF Snauwaert K, Snauwaert JF - Yoshihara H Yoshihara K Fau - Scheers, L. Scheers H Fau - Godderis, Godderis P, L Fau - Hoet, B. Hoet P Fau - Van Meerbeek, B Van Meerbeek, How much do resin-based dental materials release? A meta-analytical approach, Dent Mater 27(8) (2011) 723-747.

5. Inamitsu H, Okamoto K, Sakai E, Nishishita K, Murata H, Tsukuba T. The dental resin monomers HEMA and TEGDMA have inhibitory effects on osteoclast differentiation with low cytotoxicity. J Appl Toxicol. 2017;37(7):817-24.

6. Zhu L, Zhang J, Xiao L, Liu S, Yu J, Chen W, Zhang X, Peng B. Autophagy in resin monomer-initiated toxicity of dental mesenchymal cells: a novel therapeutic target of $\mathrm{N}$-acetyl cysteine. J Mater Chem B. 2015;3(33):6820-36.

7. Gallorini M, Cataldi A, di Giacomo V. HEMA-induced cytotoxicity: oxidative stress, genotoxicity and apoptosis. Int Endod J. 2014;47(9):813-8.

8. Oncel Torun Z, Torun D, Baykal B, Oztuna A, Yesildal F, Avcu F. Effects of triethylene glycol dimethacrylate (TEGDMA) on the odontoclastic differentiation ability of human dental pulp cells. J Appl Oral Sci. 2017;25(6):631-40.

9. Huang S, Zheng B, Jin X, Yu Q, Zhang X, Sun X, Chen Y, Ren X, Wismeijer D, Ma J, Zhang C, Wu G, Pan Y. Blockade of Cyclophilin D Attenuates Oxidative Stress-Induced Cell Death in Human Dental Pulp Cells. Oxid Med Cell Longev. 2019;4:1729013.

10. Gp. CJE,M. B. A., K. T., C. CJ., S. LJ., O. LM., G. DR., Sphingolipid metabolism cooperates with BAK and BAX to promote the mitochondrial pathway of apoptosis. Cell. 2012;148(5):988-1000. 
11. Buck MD, O'Sullivan D, Klein Geltink RI, Curtis JD, Chang CH, Sanin DE, Qiu J, Kretz O, Braas D, van der Windt GJ, Chen Q, Huang SC, O'Neill CM, Edelson BT, Pearce EJ, Sesaki H, Huber TB, Rambold AS, Pearce EL. Mitochondrial Dynamics Controls T Cell Fate through Metabolic Programming. Cell. 2016;166(1):63-76.

12. Go YM, Fernandes J, Hu X, Uppal K, Jones DP. Mitochondrial network responses in oxidative physiology and disease. Free Radic Biol Med. 2018;116:31-40.

13. Martins CA, Leyhausen G, Geurtsen W, Volk J. Intracellular glutathione: a main factor in TEGDMAinduced cytotoxicity? Dent Mater. 2012;28(4):442-8.

14. Volk J, Leyhausen G, Dogan S, Geurtsen W. Additive effects of TEGDMA and hydrogenperoxide on the cellular glutathione content of human gingival fibroblasts. Dent Mater. 2007;23(8):921-6.

15. Sb H, Qh JX,Y, Xr Z, Bb Z, Kh W, Xy S, Yt C, Xr R, Jf M, Yh WG,P. The vicious circle between mitochondrial oxidative stress and dynamic abnormality mediates triethylene glycol dimethacrylateinduced preodontoblast apoptosis. Free Radic Biol Med. 2019;134:644-56.

16. Whitmarsh AJ, Davis RJ. Multisite phosphorylation by MAPK. Science. 2016;354(6309):179-80.

17. Gehart H, Kumpf S, Ittner A, Ricci R. MAPK signalling in cellular metabolism: stress or wellness? EMBO Rep. 2010;11(11):834-40.

18. Javadov S, Jang S, Agostini B. Crosstalk between mitogen-activated protein kinases and mitochondria in cardiac diseases: therapeutic perspectives. Pharmacol Ther. 2014;144(2):202-25.

19. Park JH, Ko J, Park YS, Park J, Hwang J, Koh HC. Clearance of Damaged Mitochondria Through PINK1 Stabilization by JNK and ERK MAPK Signaling in Chlorpyrifos-Treated Neuroblastoma Cells. Mol Neurobiol. 2017;54(3):1844-57.

20. Zhu H, Jin Q, Li Y, Ma Q, Wang J, Li D, Zhou H, Chen Y. Melatonin protected cardiac microvascular endothelial cells against oxidative stress injury via suppression of IP3R-[Ca $\left.\left({ }^{2+}\right)\right] \mathrm{c} / \mathrm{VDAC}-\left[\mathrm{Ca}\left({ }^{2+}\right)\right] \mathrm{m}$ axis by activation of MAPK/ERK signaling pathway. Cell Stress Chaperones. 2018;23(1):101-13.

21. Krifka S, Hiller KA, Bolay C, Petzel C, Spagnuolo G, Reichl FX, Schmalz G, Schweikl H. Function of MAPK and downstream transcription factors in monomer-induced apoptosis. Biomaterials. 2012;33(3):740-50.

22. Eckhardt A, Gerstmayr N, Hiller KA, Bolay C, Waha C, Spagnuolo G, Camargo C, Schmalz G, Schweikl H. TEGDMA-induced oxidative DNA damage and activation of ATM and MAP kinases. Biomaterials. 2009;30(11):2006-14.

23. Brzezinski A. Melatonin in humans. N Engl J Med. 1997;336(3):186-95.

24. Li JG, Lin JJ, Wang ZL, Cai WK, Wang PN, Jia Q, Zhang AS, Wu GY, Zhu GX, Ni LX. Melatonin attenuates inflammation of acute pulpitis subjected to dental pulp injury. Am J Transl Res. 2015;7(1):66-78.

25. Cutando A, Gomez-Moreno G, Arana C, Munoz F, Lopez-Pena M, Stephenson J, Reiter RJ. Melatonin stimulates osteointegration of dental implants. J Pineal Res. 2008;45(2):174-9. 
26. Blasiak J, Reiter KJ,DJ,PE,SJ,RJ. Perspectives on the use of melatonin to reduce cytotoxic and genotoxic effects of methacrylate-based dental materials. J Pineal Res. 2011;51(2):157-62.

27. Jaiswal MK. Riluzole But Not Melatonin Ameliorates Acute Motor Neuron Degeneration and Moderately Inhibits SOD1-Mediated Excitotoxicity Induced Disrupted Mitochondrial $\mathrm{Ca}(2+)$ Signaling in Amyotrophic Lateral Sclerosis. Front Cell Neurosci. 2017;10:295-5.

28. Deng Q, Liu Q, Zhang H, Fan W, Li J, Kang J, He H, Huang F. Melatonin enhances hydrogen peroxideinduced apoptosis in human dental pulp cells. J Dent Sci. 2019;14(4):370-7.

29. Fernandez A, Ordonez R, Reiter RJ, Gonzalez-Gallego J, Mauriz JL. Melatonin and endoplasmic reticulum stress: relation to autophagy and apoptosis. J Pineal Res. 2015;59(3):292-307.

30. Liu Q, Fan W, He Y, Zhang F, Guan X, Deng Q, Lu X, He H, Huang F. Effects of melatonin on the proliferation and differentiation of human dental pulp cells. Arch Oral Biol. 2017;83:33-9.

31. Cho YA, Noh K, Jue SS, Lee SY, Kim EC. Melatonin promotes hepatic differentiation of human dental pulp stem cells: clinical implications for the prevention of liver fibrosis. J Pineal Res. 2015;58(1):127-35.

32. Mayo JC, Hevia D, Quiros-Gonzalez I, Rodriguez-Garcia A, Gonzalez-Menendez P, Cepas V, GonzalezPola I, Sainz RM. IGFBP3 and MAPK/ERK signaling mediates melatonin-induced antitumor activity in prostate cancer, J Pineal Res 62(1) (2017).

33. Navarro-Yepes J, Burns M, Anandhan A, Khalimonchuk O, del Razo LM, Quintanilla-Vega B, Pappa A, Panayiotidis MI, Franco R. Oxidative stress, redox signaling, and autophagy: cell death versus survival. Antioxid Redox Signal. 2014;21(1):66-85.

34. Sun X, Mao Y, Dai P, Li X, Gu W, Wang H, Wu G, Ma J, Huang S. Mitochondrial dysfunction is involved in the aggravation of periodontitis by diabetes. J Clin Periodontol. 2017;44(5):463-71.

35. Mikulas K, Hermann P, Gera I, Komlodi T, Horvath G, Ambrus A, Tretter L. Triethylene glycol dimethacrylate impairs bioenergetic functions and induces oxidative stress in mitochondria via inhibiting respiratory Complex I. Dent Mater. 2018;34(7):e166-81.

36. Galano A, Reiter RJ. Melatonin and its metabolites vs oxidative stress: From individual actions to collective protection. J Pineal Res. 2018;65(1):e12514.

37. Najeeb S, Khurshid Z, Zohaib S, Zafar MS. Therapeutic potential of melatonin in oral medicine and periodontology. Kaohsiung J Med Sci. 2016;32(8):391-6.

38. Tan DX, Manchester LC, Qin L, Reiter RJ. Melatonin: A Mitochondrial Targeting Molecule Involving Mitochondrial Protection and Dynamics, Int J Mol Sci 17(12) (2016).

39. Wongprayoon P, Govitrapong P. Melatonin as a mitochondrial protector in neurodegenerative diseases. Cell Mol Life Sci. 2017;74(21):3999-4014.

40. Liu J, Clough SJ, Hutchinson AJ, Adamah-Biassi EB, Popovska-Gorevski M, Dubocovich ML. MT1 and MT2 Melatonin Receptors: A Therapeutic Perspective. Annu Rev Pharmacol Toxicol. 2016;56:361-83. 
41. Zlotos DP, Jockers R, Cecon E, Rivara S, Witt-Enderby PA. MT1 and MT2 melatonin receptors: ligands, models, oligomers, and therapeutic potential. J Med Chem. 2014;57(8):3161-85.

42. Cui P, Yu M, Luo Z, Dai M, Han J, Xiu R, Yang Z. Intracellular signaling pathways involved in cell growth inhibition of human umbilical vein endothelial cells by melatonin. $J$ Pineal Res. 2008;44(1):107-14.

43. Maria S, Samsonraj RM, Munmun F, Glas J, Silvestros M, Kotlarczyk MP, Rylands R, Dudakovic A, van Wijnen AJ, Enderby LT, Lassila H, Dodda B, Davis VL, Balk J, Burow M, Bunnell BA. P.A. Witt-Enderby, Biological effects of melatonin on osteoblast/osteoclast cocultures, bone, and quality of life: Implications of a role for MT2 melatonin receptors, MEK1/2, and MEK5 in melatonin-mediated osteoblastogenesis, J Pineal Res 64(3) (2018).

44. Knani L, Bartolini D, Kechiche S, Tortoioli C, Murdolo G, Moretti M, Messaoudi I, Reiter RJ, Galli F. Melatonin prevents cadmium-induced bone damage: First evidence on an improved osteogenic/adipogenic differentiation balance of mesenchymal stem cells as underlying mechanism. J Pineal Res. 2019;67(3):e12597.

45. Dhanasekaran DN, Reddy EP. JNK signaling in apoptosis. Oncogene. 2008;27(48):6245-51.

46. Latchoumycandane C, Boelsterli SQ,TR,SJ,UA. Leflunomide or A77 1726 protect from acetaminophen-induced cell injury through inhibition of JNK-mediated mitochondrial permeability transition in immortalized human hepatocytes. Toxicol Appl Pharmacol. 2006;217(1):125-33.

\section{Figures}



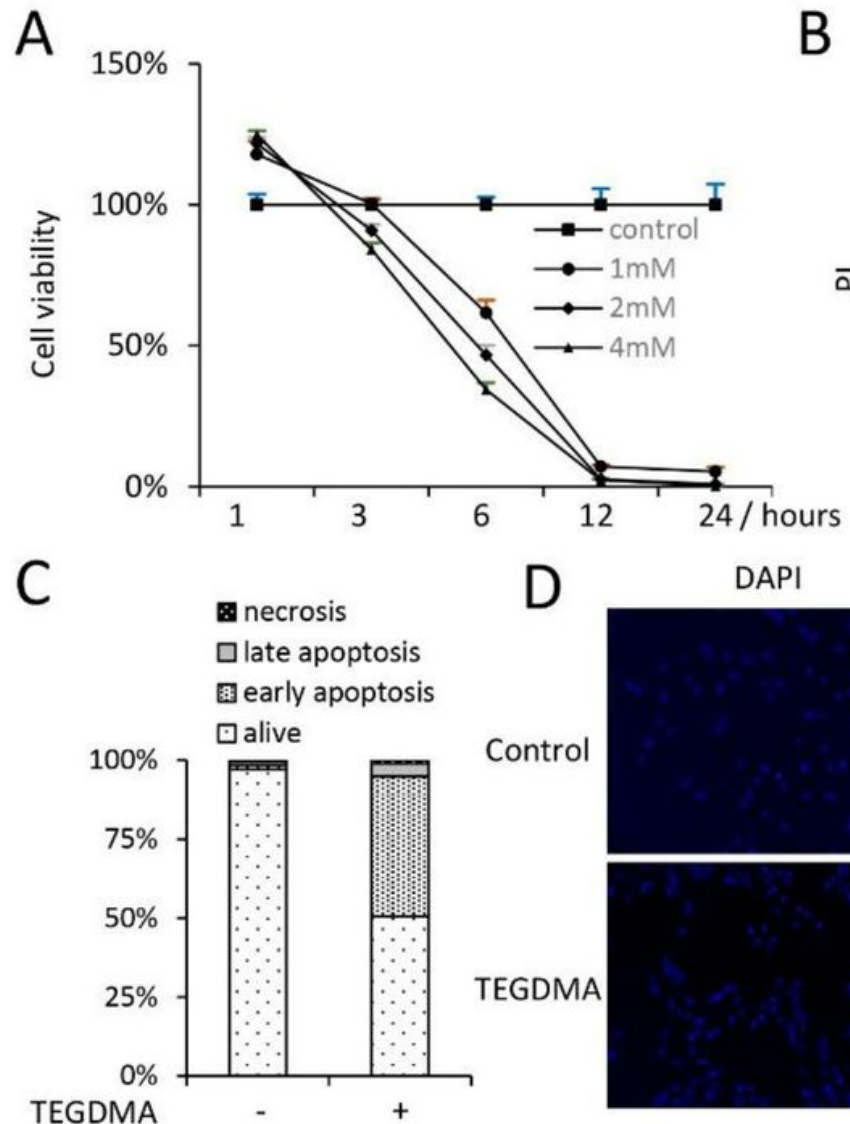

$\mathrm{F}$

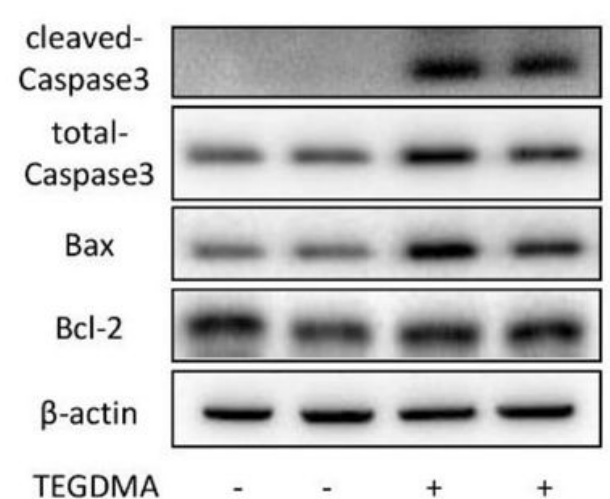

B

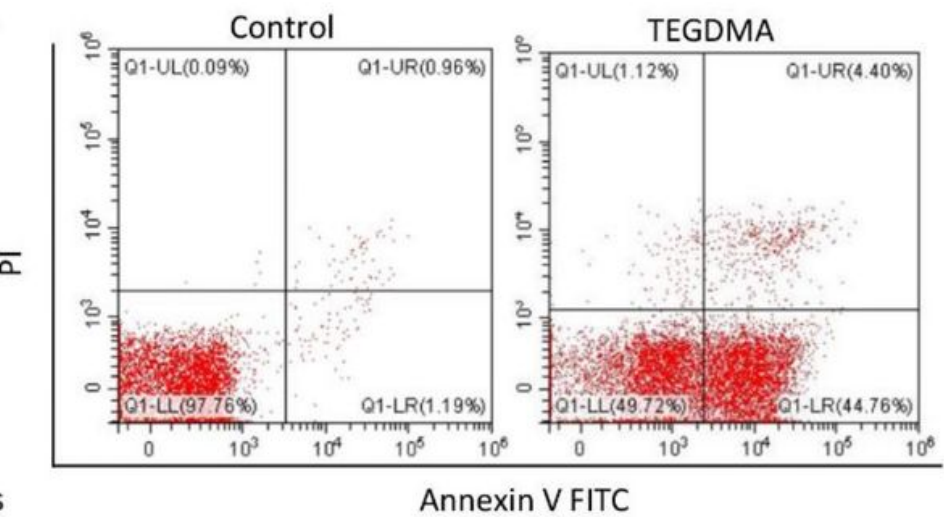

$\mathrm{E}$

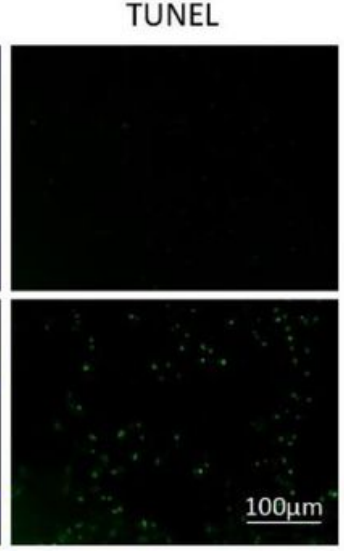

$\mathrm{H}$
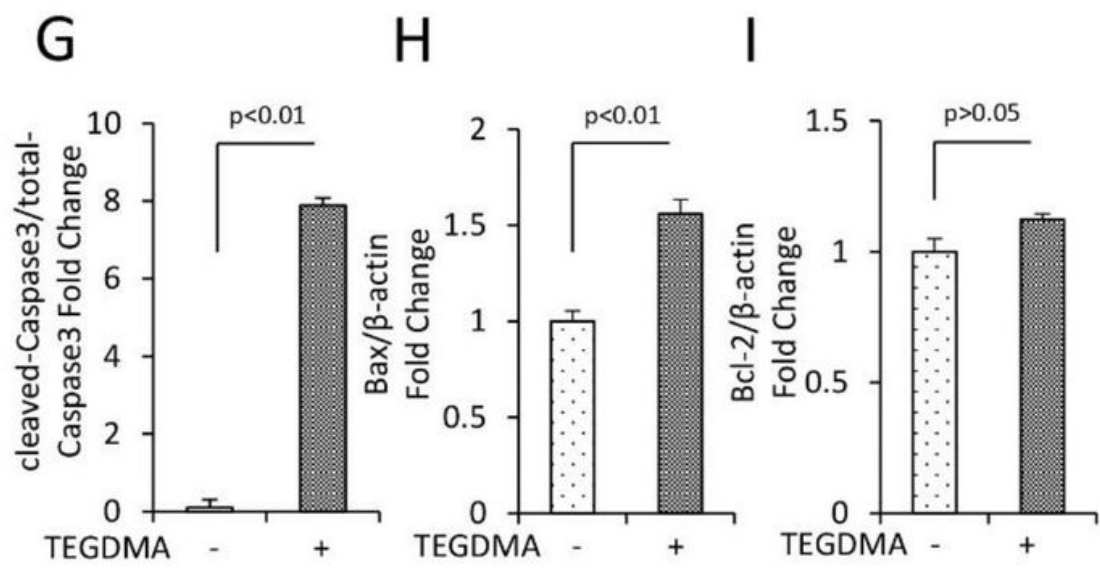

\section{Figure 1}

TEGDMA reduced cell viability and induced apoptosis in mDPC6T. (A) Cell viability was determined by MTT reduction in the mDPC6T cells with or without the presence of TEGDMA. Error bars indicate SD $(n=3)$. (B) and (C) Flow cytometric quantification of apoptosis. Error bars indicate SD $(n=3)$. (D) and (E) TUNEL staining and assay. Error bars indicate SD $(n=3)$. Western blot analysis ( $F)$ of Bax, Bcl-2, cleaved Caspase-3, and total-Caspase-3 in the presence of TEGDMA treated mDPC6T cells. Quantification of immunoreactive bands for cleaved-Caspase3 (G) relative total-Caspase3, Bax (H) and Bcl-2 (I) relative to $\beta$-actin, Error bars indicate SD $(n=3)$. 


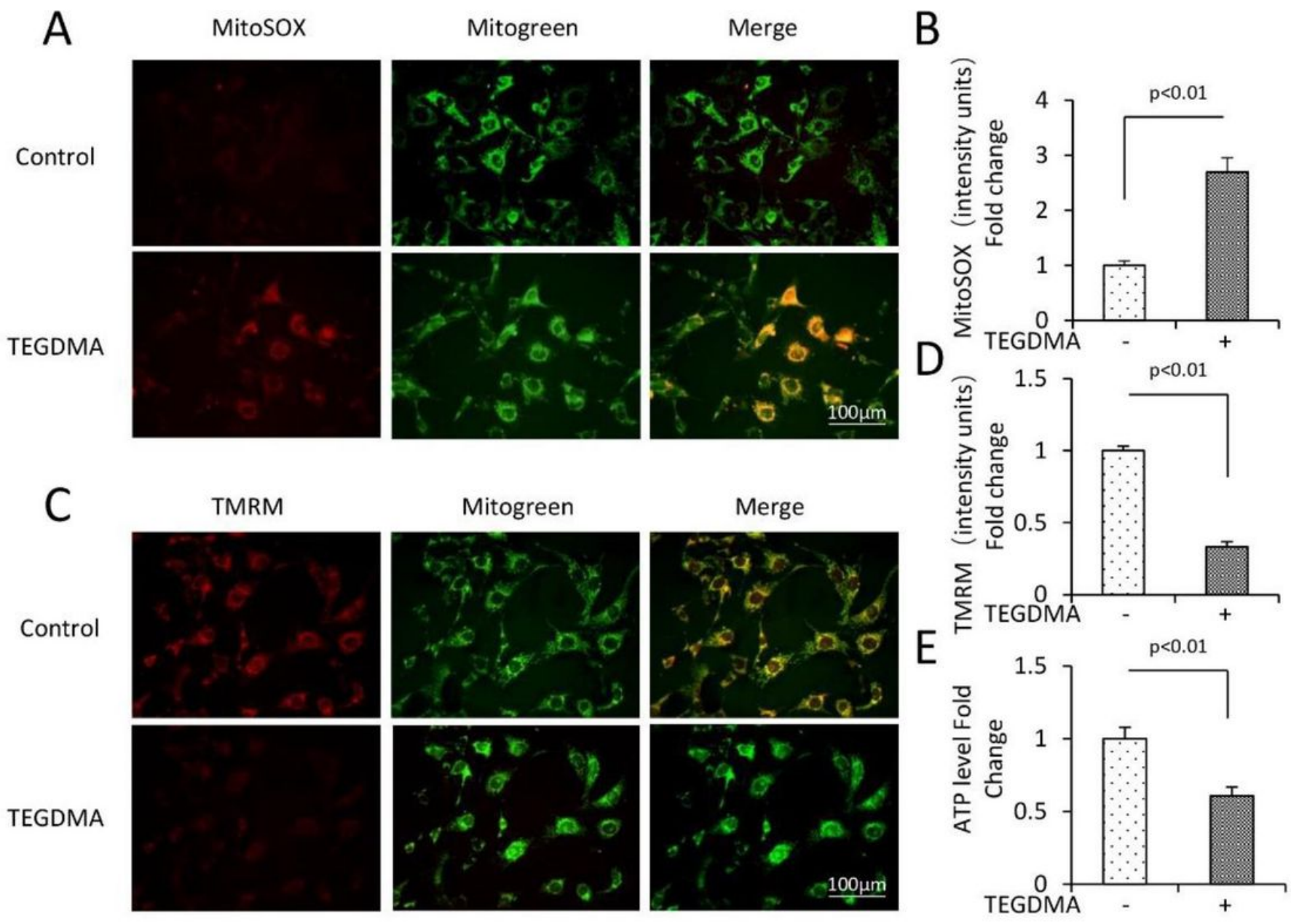

Figure 2

TEGDMA-induced mitochondrial dysfunction in mDPC6T cells. (A) and (B) Representative images showing MitoSOX staining and quantification in the indicated groups $(n=3)$. (C) and (D) Representative images with TMRM staining and quantification in the indicated groups $(n=3)$. Cellular ATP level $(M)$ in the indicated groups $(n=3)$. 


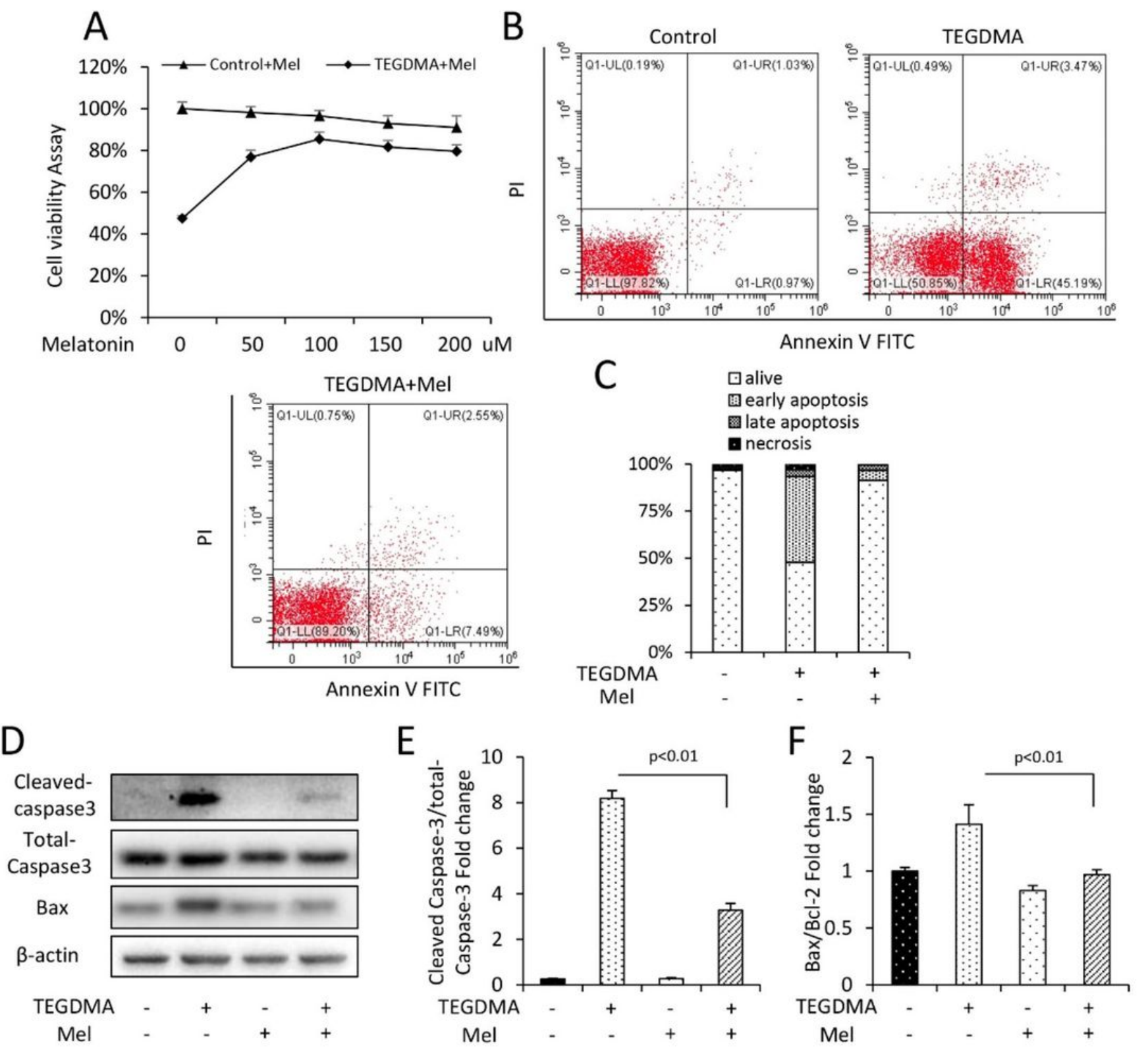

\section{Figure 3}

Melatonin attenuated TEGDMA-induced apoptosis in mDPC6T cells. (A) Cell viability determined by MTT reduction in mDPC6T cells in the presence of melatonin (Mel) with or without TEGDMA. Error bars indicate SD ( $n=3)$. Flow cytometry (B) and (C) assay after melatonin treatment. Error bars indicate SD $(n=3)$. (D) Representative immunoreactive bands for cleaved-Caspase3 and Bax in mDPC6T cells with (+) or without (-) melatonin treatment in the presence of TEGDMA (+) or culture medium (-). Quantification of immunoreactive bands for cleaved-Caspase3 (E) relative to total-Caspase3, Quantification of immunoreactive bands for $\operatorname{Bax}(F)$ relative to $\beta$-actin. Error bars indicate $S D(n=3)$. 


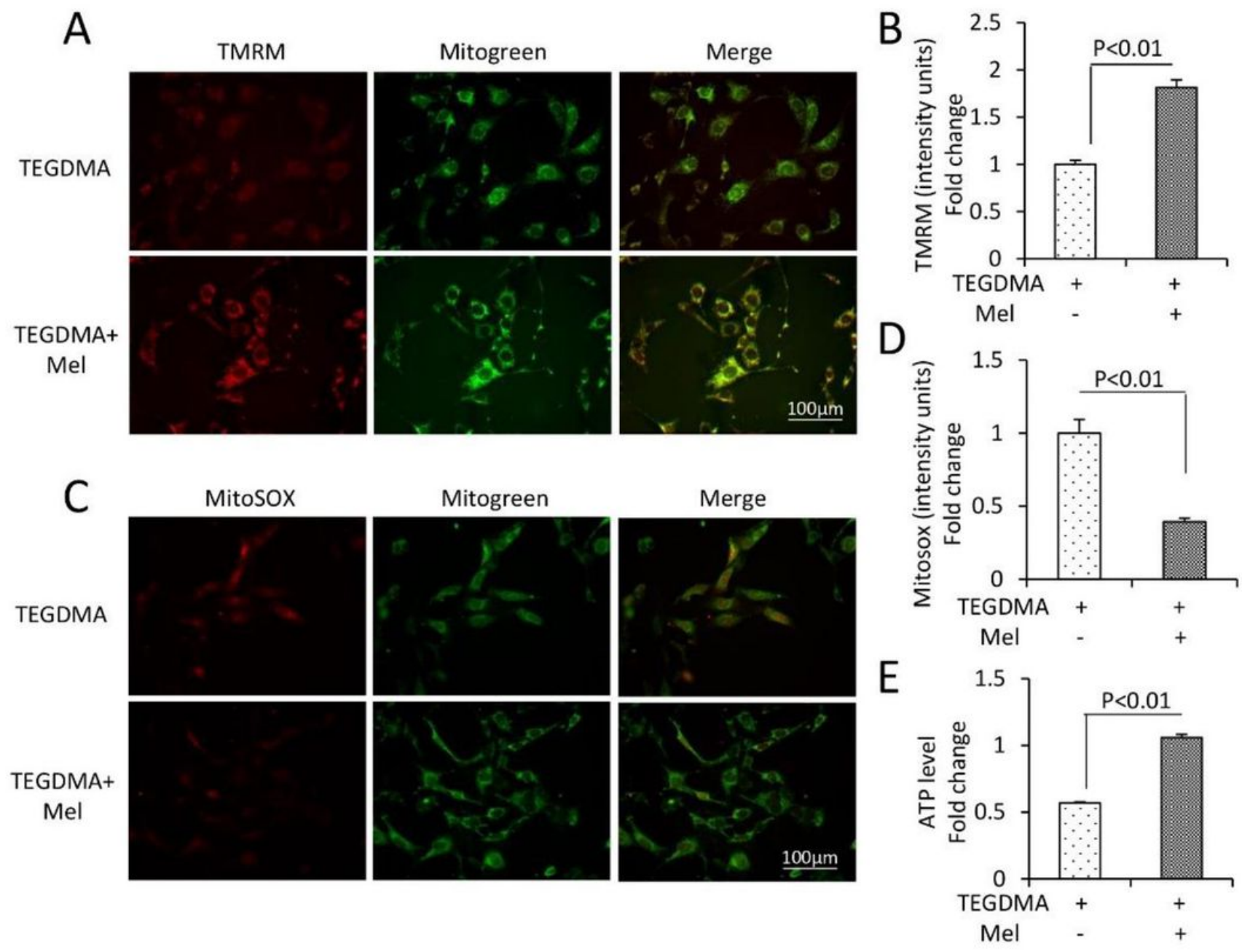

Figure 4

Melatonin ameliorated TEGDMA-induced mitochondrial dysfunction in mDPC6T cells. Representative images showing TMRM staining (A) and quantification (B) in the indicated groups $(n=3)$. Representative images with MitoSOX staining (C) and quantification (D) in the indicated groups $(n=3)$. Cellular ATP level $(E)$ in the indicated groups $(n=3)$. 


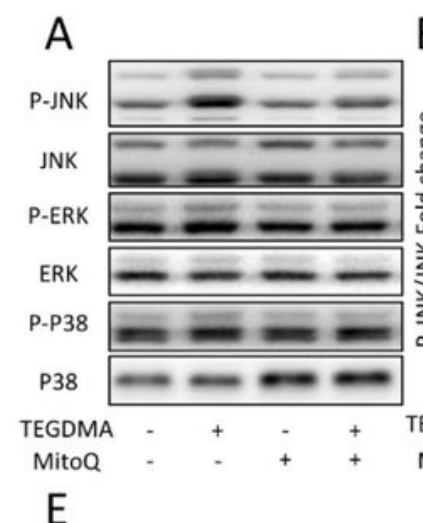

B

C

D
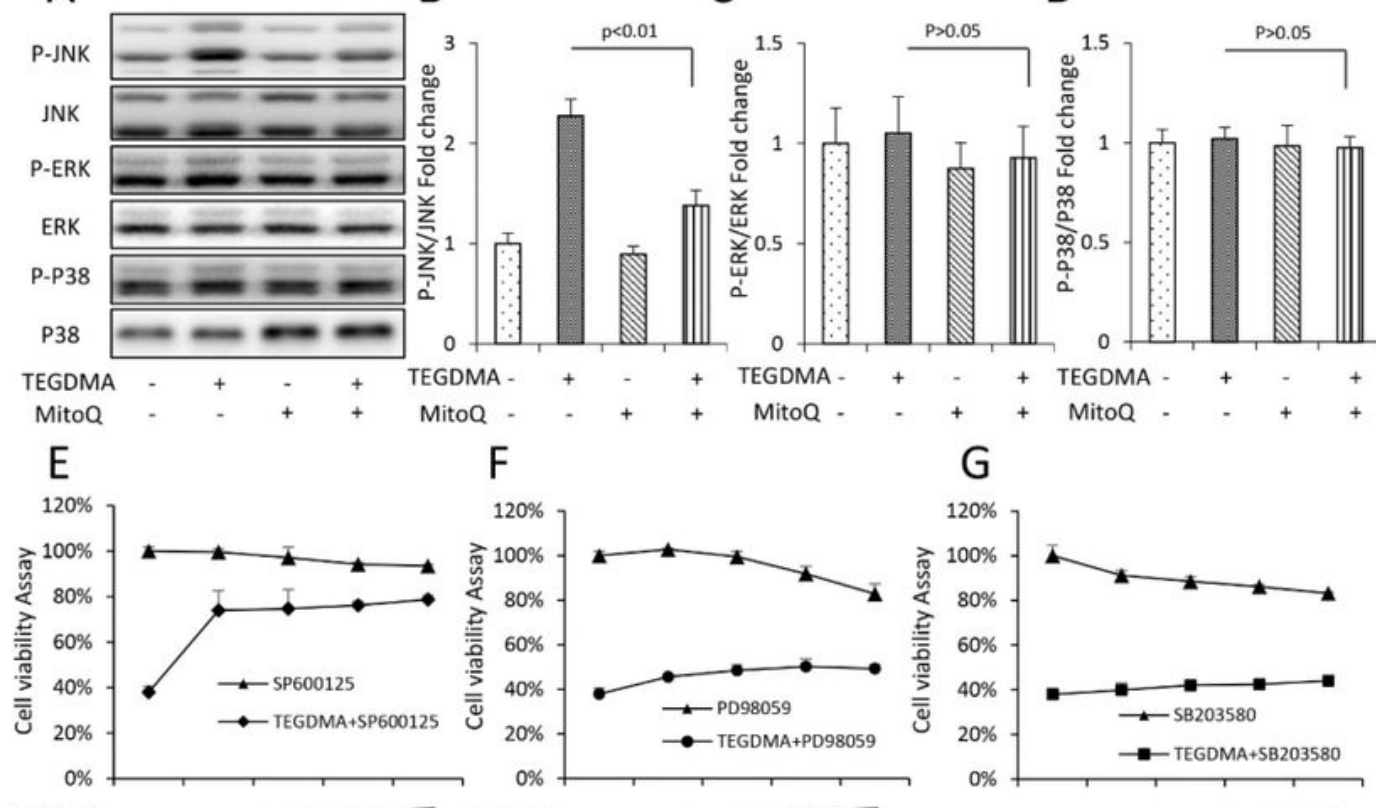
TEGDMA itoQ

$\mathrm{F}$

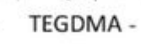

+ TEGDMA

MitoQ -
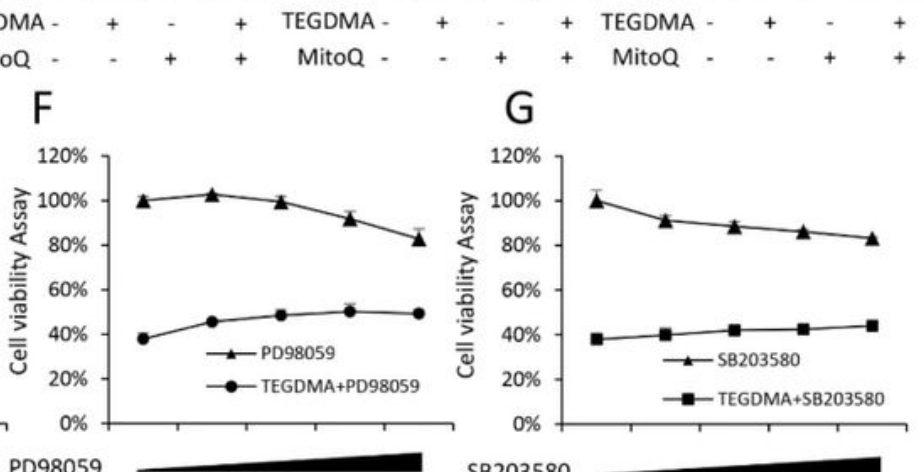

SP600125

PD98059

$\mathrm{H}$
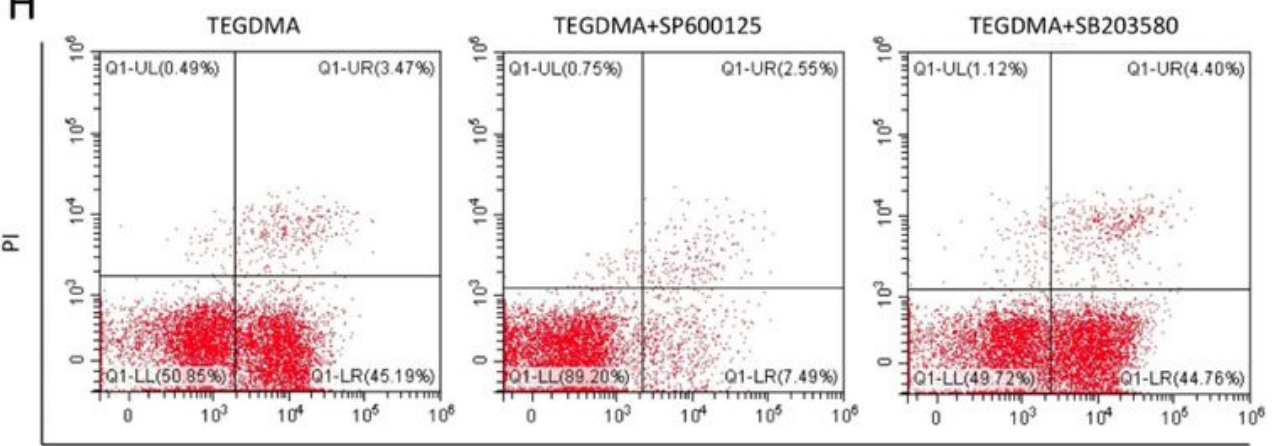

Annexin V FITC
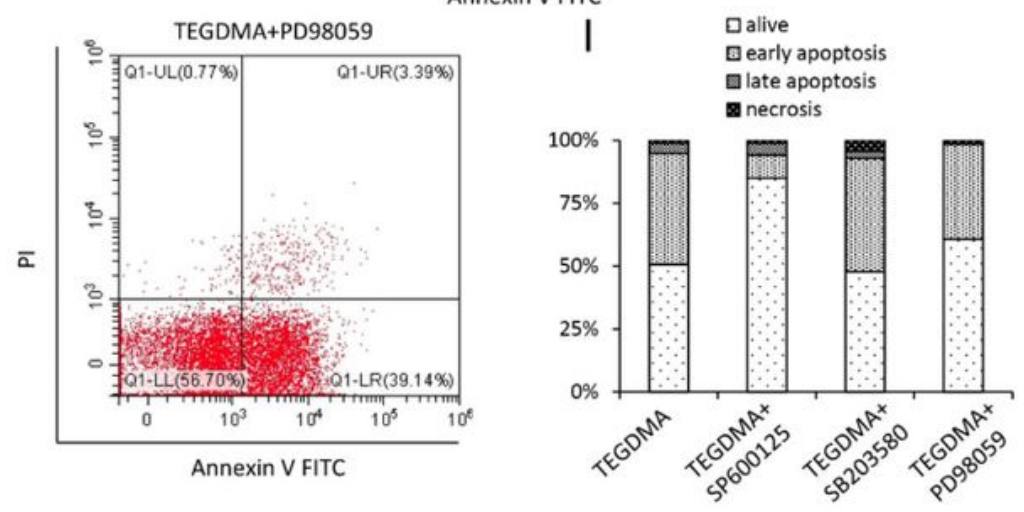

\section{Figure 5}

The role of MAPKs pathway involved in TEGDMA induced mDPC6T apoptosis. (A) Representative immunoreactive bands for MAPKs in mDPC6T cells with (+) or without (-) MitoQ treatment in the presence of TEGDMA (+) or culture medium (-). (B) Quantification of immunoreactive bands for p-JNK relative to JNK. (C) Quantification of immunoreactive bands for $p$-ERK relative to ERK. (D) Quantification 
of immunoreactive bands for $\mathrm{p}-\mathrm{p} 38$ relative to $\mathrm{p} 38$. Error bars indicate SD $(\mathrm{n}=3)$. Flow cytometry $(H)$ and (I) assay after TEGDMA with or without MAPKs inhibitors treatment. Error bars indicate SD $(n=3)$.

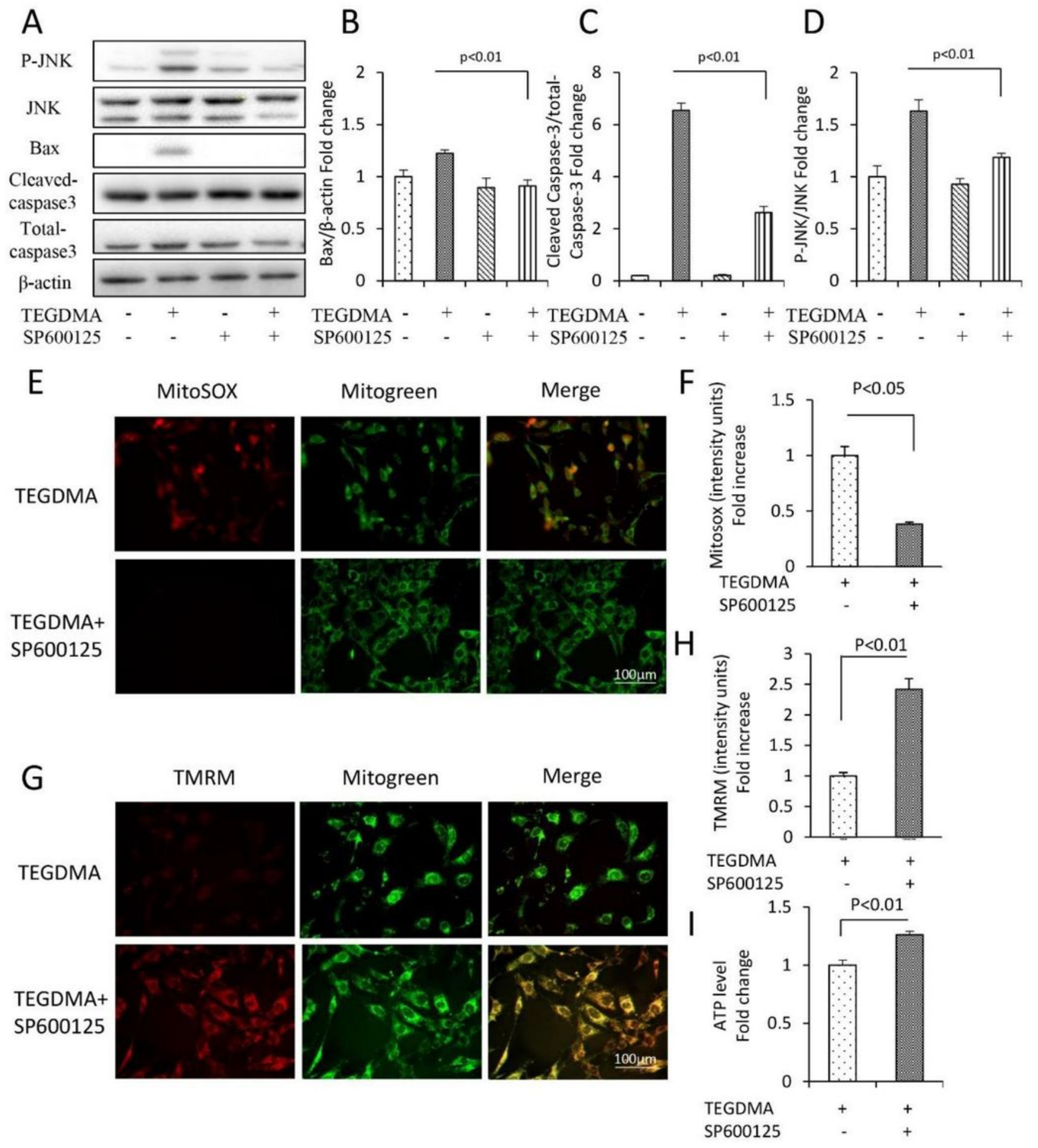

Figure 6

The role of JNK/MAPK pathway on TEGDMA induced mDPC6T apoptosis and mitochondrial dysfunction. (A) Representative immunoreactive bands in mDPC6T cells with (+) or without (-) SP600125 treatment in the presence of TEGDMA (+) or culture medium (-). (B) Quantification of immunoreactive 
bands for Bax relative to $\beta$-actin. (C) Quantification of immunoreactive bands for cleaved-Caspase3 relative to total-Caspase3. (D) Quantification of immunoreactive bands for $p$-JNK relative to JNK. Error bars indicate SD $(n=3)$. Representative images show MitoSOX staining $(E)$ and quantification $(F)$ in the indicated groups $(n=3)$. Representative images with TMRM staining $(G)$ and quantification $(H)$ in the indicated groups $(n=3)$. Cellular ATP level $(I)$ in the indicated groups $(n=3)$.
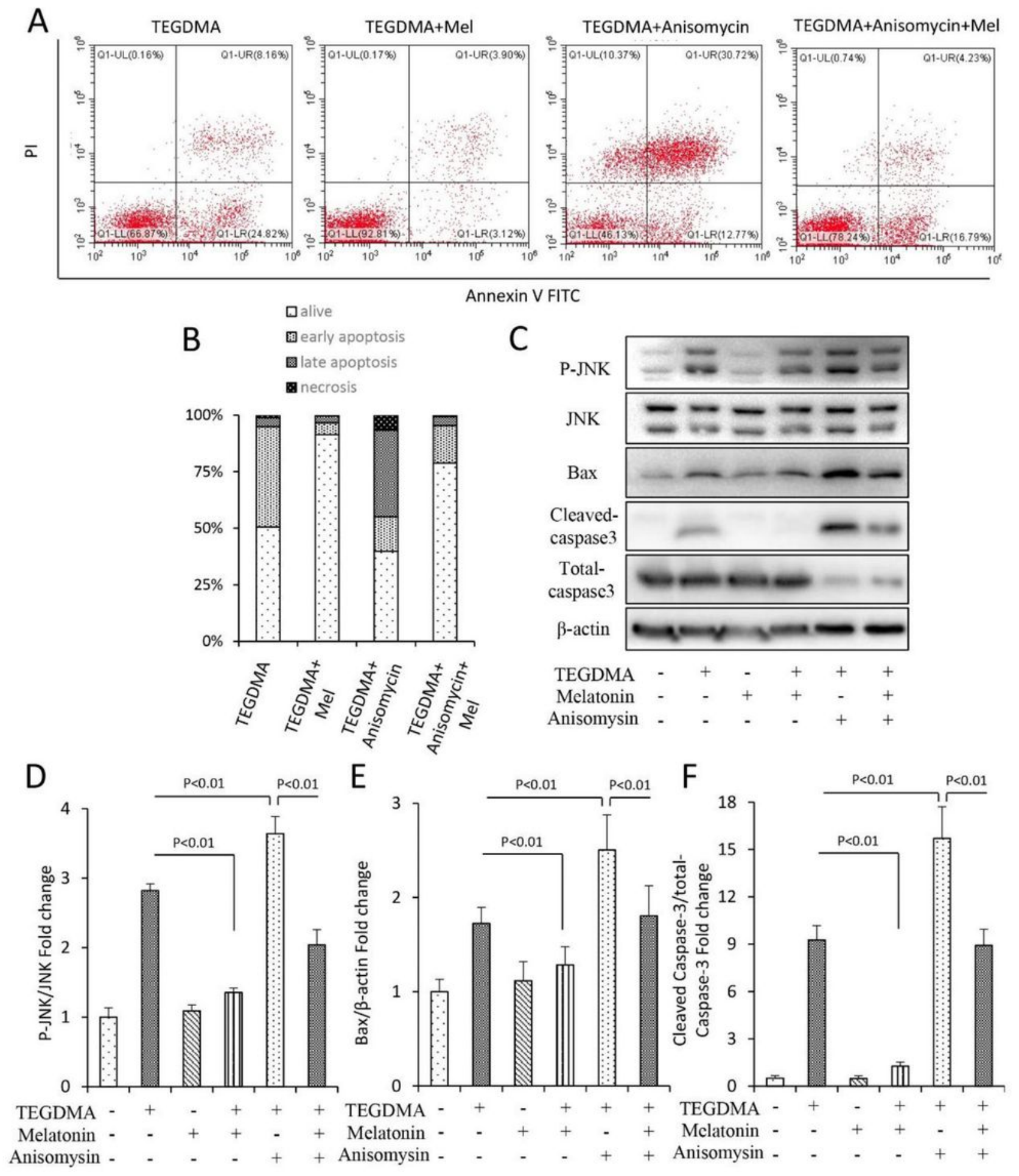

Figure 7 
Melatonin prevented TEGDMA-induced apoptosis through JNK/MAPK pathway. Flow cytometry (A) and assay (B) after TEGDMA with or without melatonin under the presence of JNK/MAPK agonist Anisomycin, Error bars indicate SD ( $n=3)$. (C) Representative immunoreactive bands in mDPC6T cells with (+) or without (-) melatonin and Anisomycin in the presence of TEGDMA (+) or culture medium (-). (D) Quantification of immunoreactive bands for p-JNK relative to JNK. (E) Quantification of immunoreactive bands for Bax relative to $\beta$-actin. (F) Quantification of immunoreactive bands for cleaved-Caspase3 relative to total-Caspase3. Error bars indicate SD $(n=3)$.

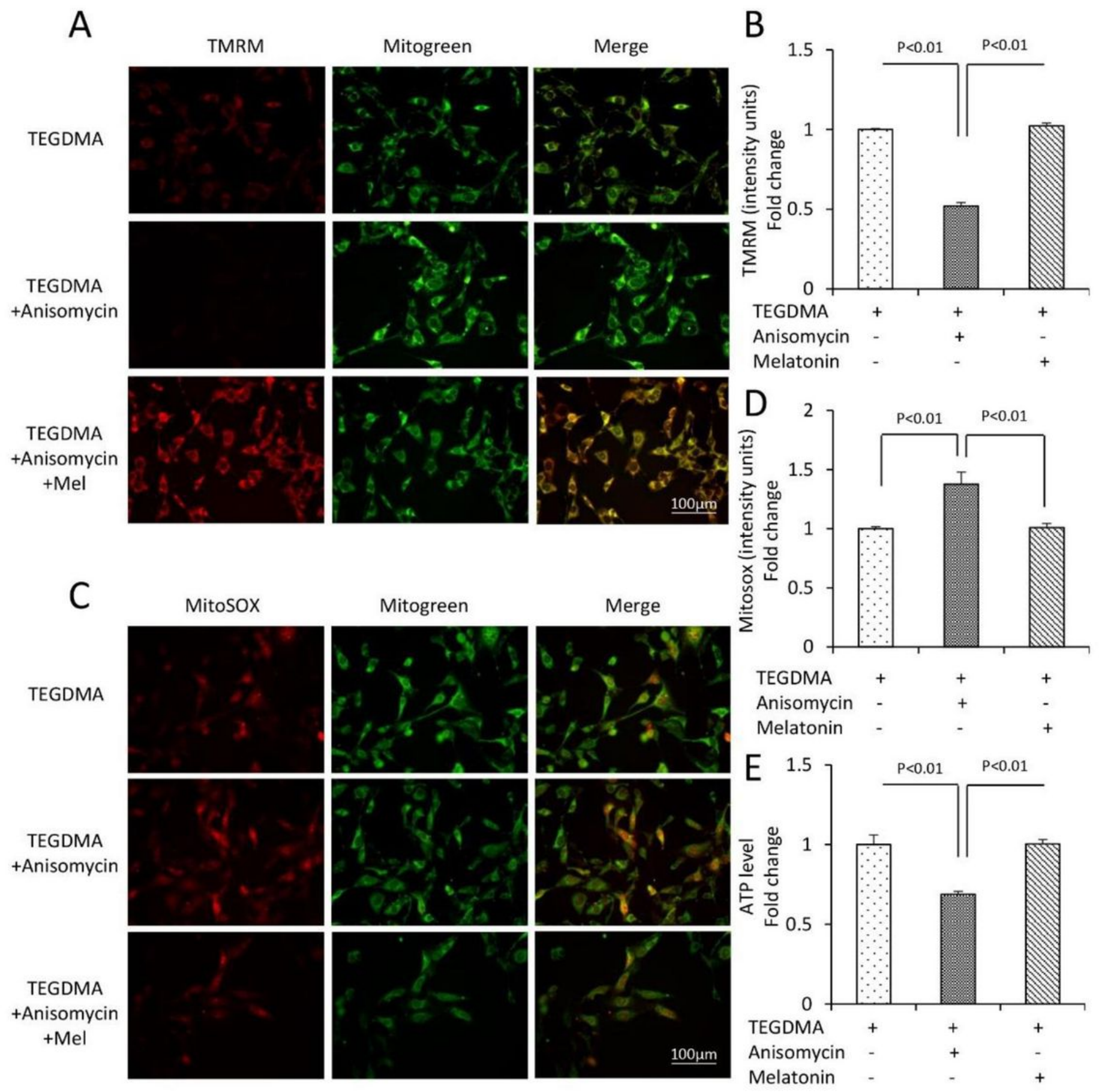

Figure 8 
Melatonin attenuated TEGDMA-induced mDPC6T mitochondrial dysfunction through JNK/MAPK pathway. Representative images showing TMRM staining (A) and quantification (B) in the indicated groups $(n=3)$. Representative images with MitoSOX staining (C) and quantification (D) in the indicated groups $(n=3)$. Cellular ATP level $(E)$ in the indicated groups $(n=3)$.

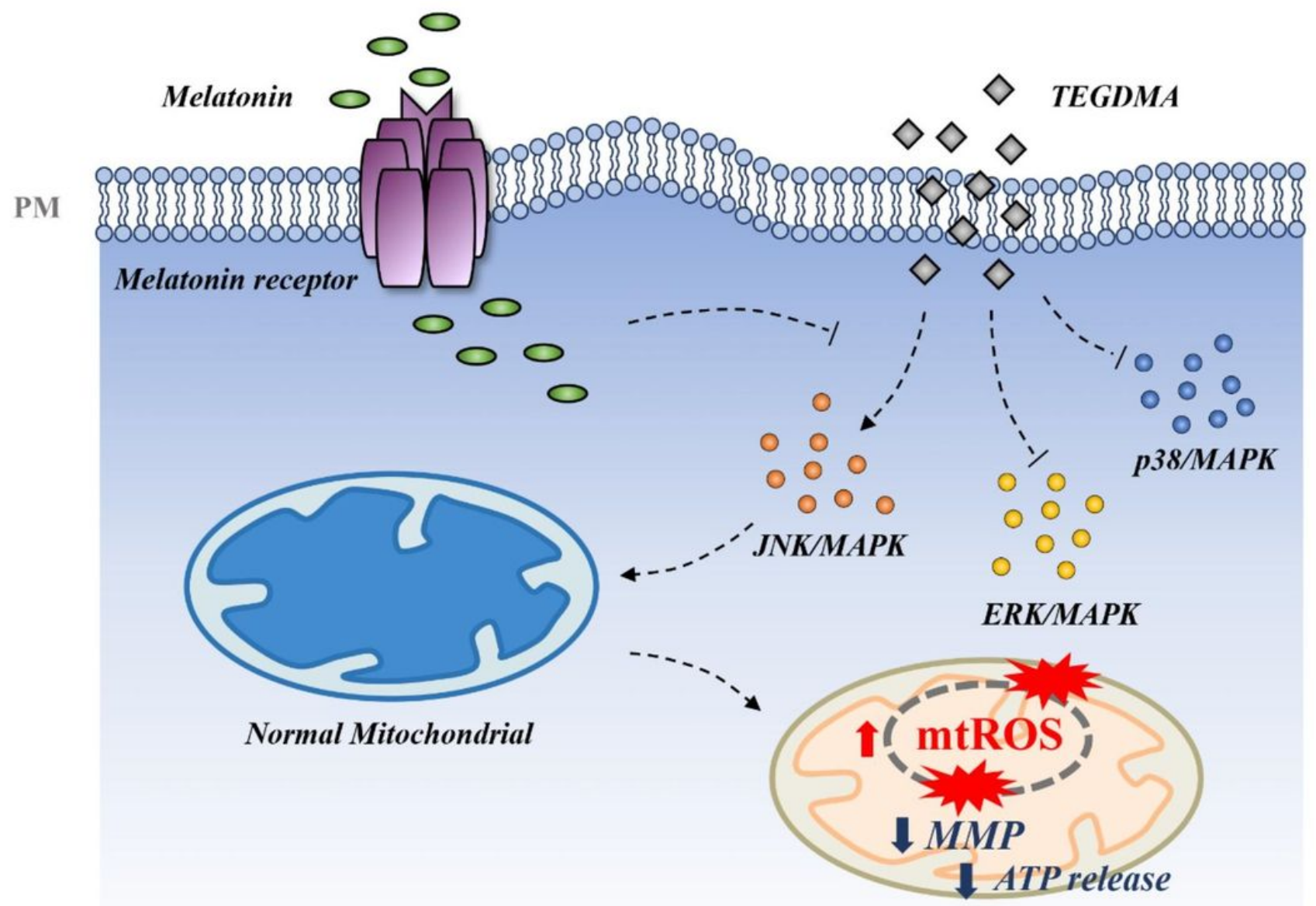

Cytosol

Dysfunction Mitochondrial

Figure 9

Graphical abstract

Apoptosis 